\title{
Sub-culturas en la Escuela: Análisis de sus contenidos y relaciones
}

\author{
Mario Sandoval M*
}

\begin{abstract}
Resumen
El artículo que se presenta a continuación da cuenta de las complejas relaciones entre escuela, subculturas y juventud. En esta tríada se conjuga un conjunto de aspectos que hacen referencia a los procesos pedagógicos, a la función docente y a las clásicas funciones que cumple la escuela, a saber, la certificación del conocimiento, la función educativa propiamente tal y la función de socialización. En síntesis, se plantea que de no haber un reconocimiento explícito de las subculturas juveniles al interior de los espacios escolares no se podrá desarrollar un proceso pedagógico en toda su potencialidad.
\end{abstract}

Palabras clave: jóvenes, estudiantes, cultura

\begin{abstract}
The present article accounts for the complex relationships between school, subcultures and youth. In this triad, a set of aspects combined make reference to pedagogical processes, teacher's role and the typical functions that the school performs; namely, the certification of knowledge, the educational function as such, and the role of socialization. In a nutshell, it is considered that, without having an explicit recognition of the youthful subcultures within the school premises, a full and powerful pedagogical process will hardly take place.
\end{abstract}

Key words: young people, students, culture

El presente artículo es producto del Proyecto Fondecyt N N 1070105 “Cultura Juvenil y producción valórica en estudiantes de educación secundaria y educación superior".

* Dr. en Sociología Université Catholique de Louvain, Coordinador del Centro de Estudios en Juventud (CEJU) de la Universidad Católica Silva Henríquez. msandoval@ucsh.cl 


\section{Introducción}

La relación escuela-sociedad es de larga data y contiene en su seno un conjunto de elementos que hacen referencia al desarrollo de los pueblos. Es así como John Dewey (1989) hace hincapié en el hecho de que la escuela para ser efectiva tiene que ser una institución íntimamente relacionada con la sociedad. Sin embargo, en esta relación polar interviene otro factor esencial, sin el cual no se puede comprender su dinámica y desarrollo; nos referimos a los jóvenes, los que comúnmente son tipificados como "alumnos" desconociendo o no queriendo reconocer la complejidad del fenómeno juvenil que se introduce en la escuela, generando procesos complejos que muchas veces no son comprendidos por los educadores dando lugar a una relación tensa y conflictiva entre profesores y alumnos.

En este sentido, pareciera ser que subyace en la visión del magisterio la imagen de una juventud irresponsable, apolítica, descreída, altamente influenciable, etc., es decir, la imagen de una juventud conflictiva, llena de carencias y fallas que la escuela debe corregir.

Estos estereotipos sociales y culturales cubren la diversidad juvenil que se manifiesta en los espacios escolares dando origen a numerosas subculturas, las cuales, muchas veces, buscan canales de expresión reñidos con la institucionalidad vigente o en contraposición a los reglamentos que rigen y caracterizan a los espacios escolares.

Es así como se genera una tensión generacional entre educadores y alumnos, entre adultos y jóvenes. Esta relación asimétrica que tiene una expresión simbólica se manifiesta de manera clara y transparente y otras veces utiliza canales de expresión soterrados, ocultos o invisibles (bullings, por ejemplo).

En esta perspectiva la escuela podría / debería jugar un rol en la formación de la ciudadanía juvenil contribuyendo con un 
mejor desarrollo de cada uno de los sujetos que participan en su interior. Un proyecto de esta naturaleza debe contemplar asuntos relacionados con las finalidades de la educación, con los planes de estudio, con las políticas del sistema educativo en general y de las instituciones educativas en particular, con las formas didácticas y con el desarrollo de las prácticas y relaciones interpersonales.

Estos asuntos se derivan de los puntos de vista que tengan dichos actores acerca del tipo de sujeto que se desea contribuir a formar y de la sociedad que se quiere hacer realidad.

De estos temas trata el presente Ensayo; se trata de dar cuenta del fenómeno de las subculturas al interior de los espacios escolares; cómo esas subculturas se manifiestan y qué relación tienen con los sujetos que las protagonizan, todo ello en un marco regulatorio que deja pocos espacios para la curiosidad, para la inventiva, para la creatividad y la sorpresa propias de las subculturas juveniles.

\section{Aproximación conceptual del fenómeno juvenil}

En primer lugar daremos cuenta del concepto central que subyace a las subculturas escolares, nos referimos al concepto de juventud, nos ha parecido necesario hacerlo puesto que son ellos (los jóvenes) quienes le dan vida a las subculturas al interior de la escuela.

Al utilizar el concepto "juventud", "período Juvenil" o "adolescencia", pareciera ser que todos se están refiriendo a lo mismo, sin embargo, constatamos que la categoría juvenil es muy diversa teórica y empíricamente. En el campo teórico las diferencias están marcadas por las distintas corrientes psico-sociológicas que se preocupan del tema, en el campo empírico es posible advertir diferencias según el lugar geográfico donde se viva, la época histórica, la pertenencia a un determinado sector social, las características de la cultura imperante, etc. 
Es así como podemos aproximarnos al fenómeno juvenil desde tres perspectivas, estas son:

- La juventud como categoría etaria: Esta es una perspectiva sociodemográfica y coloca el énfasis en la condición etaria, por lo que se define como jóvenes a todas aquellas personas que tiene entre 15 y 24 años de edad (Naciones Unidas, 1983). Cabe destacar que en Chile la población juvenil se encuentra definida por el Instituto Nacional de la Juventud (INJUV) como aquellas personas que se encuentra entre los 15 y 29 años de edad. Según el último Censo, hay 3.648.000 jóvenes en nuestro país.

Al término de la juventud, esta masa de jóvenes debería insertarse en el mundo adulto, dicha inserción se logra, entre otras cosas mediante la obtención de un trabajo estable y la fundación de una familia la cual posibilita ocupar una posición específica en el juego de roles y status en la sociedad.

- La juventud como etapa de maduración: Esta perspectiva de análisis coloca el énfasis en los procesos ligados a los cambios fisiológicos y psicológicos que repercuten en la forma de ser joven. En este campo, se juega el gran tema de la identidad juvenil y existe acuerdo entre los expertos en señalar que las áreas de la afectividad, sexualidad, social, intelectual y físico-motora son fundamentales en el desarrollo integral de los jóvenes.

En el proceso de maduración, la institución del trabajo es fundamental; esta maduración sería adquirida cuando la persona está en pleno uso de sus derechos y deberes de adulto. Desde esta óptica la juventud es un período de moratoria, en la que se debe desarrollar una preparación para ingresar al mundo adulto, por lo tanto la educación juega un rol clave en la preparación de los jóvenes; en consecuencia los espacios donde se verifica el proceso educativo (las escuelas) son fundamentales para garantizar esta preparación y en un sentido más largo para formar ciudadanía juvenil. 
En el sentido anteriormente descrito es fundamental reconocer la importancia de la cultura juvenil para el trabajo formativo en la escuela, hacerlo es al mismo tiempo reconocer que los jóvenes que entran a la escuela poseen una densidad cultura propia, no son una hoja en blanco sobre la cual escriben los profesores.

Este reconocimiento explícito puede aumentar la riqueza comunicativa de los intercambios al interior del espacio escolar, dicho en otras palabras: si los profesores, escuchan, aceptan y acogen la diversidad de las subculturas juveniles podrán enriquecer los procesos educativos, reconociendo y tomando en cuenta el mundo vital de los jóvenes y a partir de ahí darle sentido a los procesos pedagógicos.

De acuerdo a lo señalado anteriormente, cabe preguntarse ¿cuándo termina la juventud? Ante esta pregunta no se pueden dar respuestas absolutas, por lo cual no se puede identificar el término de la juventud sólo con la finalización del desarrollo biológico, ni tampoco sirve la determinación legal del término de la juventud, puesto que si bien se completan ciertas áreas de desarrollo o se adquiere un determinado status legal en la sociedad, esto no significa que la persona haya logrado una inserción exitosa en el mundo social adulto.

- La juventud como subcultura: La perspectiva cultural permite comprender el fenómeno juvenil más integralmente. Esta dimensión nos permite entender a los y las jóvenes dentro de un tiempo y espacio histórico, donde el pensar y actuar son propios de un contexto determinado.

Los jóvenes chilenos, desde sus vivencias y cotidianeidad, han intentado proponer a la sociedad un conjunto de hechos culturales, constatándose una generación juvenil portadora de un conjunto de formas de ver, sentir, pensar y hacer que guían su conducta y la caracteriza, diferenciándola de otros grupos sociales. 


\section{Las subculturas en la Escuela}

Por lo anteriormente expresado, la situación de los jóvenes chilenos constituye una preocupación central desde distintos ámbitos de la sociedad porque constituyen un potencial un grupo de presión social, (al respecto véase la "revolución de los pingüinos"), porque son considerados un segmento electoral necesario en momentos de elecciones, o porque representan una masa consumidora de inmejorables proyecciones. De esta manera, los jóvenes han pasado a constituir un grupo objeto de preocupación para las autoridades políticas, sociales, educativas, religiosas y económicas de la sociedad.

Para el desarrollo de cualquier sociedad se requiere de la participación efectiva de todos los grupos que la componen. En el contexto actual de nuestro país, la participación social de los jóvenes no se ha conseguido satisfactoriamente, obstaculizando así el proceso de modernización, transición democrática y la integración social de este grupo. Considerando, que se les vincula generalmente a actitudes relacionadas con el consumo de drogas y alcohol, violencia callejera, delincuencia, apatía frente a la política; contraponiéndose todos estos aspectos con las expectativas que la institucionalidad tiene de los jóvenes.

Entonces, el gran desafío de los jóvenes chilenos es relacionarse con una sociedad y un modelo económico que los seduce a consumir y a participar de las modernizaciones, de los éxitos económicos; pero al mismo tiempo los rechaza, excluye, los ignora y/o los castiga por su condición juvenil.

De lo anterior se desprende un cruce obligado y necesario entre los jóvenes y escuela. Es esta institución el espacio privilegiado donde miles de jóvenes se educan, se socializan y se preparan para asumir sus roles de adultos de mejor manera. Desde esta perspectiva el sentido que en las instituciones educativas se debe fomentar es la formación de personas críticas, deliberantes y pensantes. 
Respecto de lo anterior cabe recordar las tres funciones básicas que cumple la escuela en relación a los jóvenes (Dubet \& Martucelli, 1998): La primera función se refiere a que la escuela atribuye calificaciones escolares que poseen cierta utilidad social en la medida que ciertos empleos, posiciones o status están reservados a los diplomados; es lo que se conoce tradicionalmente como certificación del conocimiento. La segunda es la función educativa, es decir, la producción de un tipo de sujeto adecuado a su utilidad social.

La tercera función de la escuela es la socialización, es decir, la escuela produce un tipo de individuo adaptado a la sociedad en la cual vive, en ese sentido, la escuela es un aparato de distribución de posiciones sociales, es un aparato de producción de actores ajustados a esas posiciones. En relación a esta última función es interesante observar las diferencias entre la enseñanza municipal, la particular subvencionada y la particular pagada; no es casualidad que a cada una está asociada un determinado tipo de jóvenes, portadores de subculturas específicas; es la diferencia de capital social y cultural (Bourdieu P., 2003) asociado a la familia de origen.

Barbero J. (2001) plantea algunos asuntos que están por resolverse respecto de las relaciones entre la sociedad y las nuevas generaciones. Este autor plantea que son varios los desafíos que tiene la sociedad latinoamericana con los jóvenes y a su vez los jóvenes con sus países. Estos desafíos pueden ser similares para diferentes actores sociales, en diferentes contextos, sobre todo para aquellos que participan en la vida de las instituciones escolares, lo cual puede hacer de ellas escenarios donde sea posible la formación de personas que participen activamente en el desarrollo del país.

El primer desafío se refiere a ser ciudadanos en países donde hay poco sentido de comunidad; es difícil ser ciudadano cuando se desconocen los mitos fundadores y se aprecian poco símbolos 
capaces de cohesionar un nosotros donde quepamos todos. Un segundo desafío consiste en ser ciudadanos en países desinstitucionalizados, países con precarias instituciones legales lo cual hace difícil que la gente encuentre proyectos políticos y/o éticos que respondan a sus expectativas.

El tercer desafío es ser ciudadanos en presencia de subculturas donde se privilegia el desconocimiento de la ley, se idealiza el modo de ser, de pensar y de actuar de personajes que transgreden las normas y las pautas de convivencia social y donde se acepta y se valora la cultura del "vivo", de la trampa y del engaño. En este contexto se visualiza un fenómeno preocupante en las escuelas, nos referimos a la violencia escolar, al bullings, a la irrupción de conductas desadaptativas que incomodan a los profesores y quienes dirigen los establecimientos escolares.

Muchas son las explicaciones acerca del origen de la violencia en las escuelas, lo importante en este caso es comprender el sentido de la misma, es decir, comprender qué nos quieren decir los jóvenes estudiantes a través de sus conductas violentas, qué mensaje nos quieren transmitir, que no logran comunicar de otra forma. Conocidos son los casos de expresiones violentas al interior de la escuela asociadas a las llamadas tribus urbanas (a las que nos referiremos un poco más adelante); no en pocas ocasiones los patios de las escuelas se transforman en campos de batalla entre tribus rivales, alterando la normal convivencia escolar y tensionando las relaciones entre profesores y alumnos.

Todo lo anterior se concretiza en un escenario en profundo proceso de cambio cultural. El fenómeno que se vive en el país es el desarrollo de un proceso de modernización, unido a otro proceso más largo e imperceptible a simple vista, cual sería un proceso lento de mutación cultural, que se verifica en la vida cotidiana de los jóvenes, sea cual sea el espacio sociocultural que vivan en su cotidianeidad.

Dicho de otro modo, se está produciendo un cambio cultural 
por abajo o por dentro; en los actos cotidianos, en el trabajo, en el consumo, en las relaciones de pareja, en los proyectos personales, en el uso del tiempo libre, en los grupos de amigos, en la escuela, el Liceo y/o en la Universidad, etc.

La hipótesis interpretativa de este fenómeno pertenece a Guy Bajoit y Abraham Franssen (1995). Dichos autores señalan que: "Desde hace 20 o 30 años, una mutación cultural está en curso" (Bajoit G. \& Franssen A., 1995. p.185), es decir, estaríamos viviendo el paso "de un modelo cultural basado en la razón social a otro fundado sobre la autorrealización autónoma" (Bajoit G. et al, p. 186), y más aún, "la reducción de la credibilidad que afecta al modelo de la razón social y el aumento de la credibilidad que se vincula al modelo de la autorrealización autónoma serían al final un proceso irreversible en la medida en que este sería alentado por todos, incluso por aquellos que aparentemente se esfuercen por resistirlo" (Bajoit G. et al, p. 186).

El telón de fondo del cual parten los autores señalados, es la idea que hoy día estaríamos viviendo un período de mutación cultural y que esta mutación dificulta que los jóvenes encuentren sentido a las cosas que hacen y -como consecuencia- les resulta difícil vivir.

En un mundo que les exige cada vez más, se va produciendo un desfase entre las expectativas de éxito y los límites o los obstáculos que ellos perciben en el logro de esos objetivos.

En un contexto cultural inestable, en mutación, el primer fenómeno que se produce es que las expectativas de los otros dejan de ser homogéneas y pasan a ser más o menos incoherentes; la socialización deviene paradojal. Los valores del antiguo modelo pierden su legitimidad progresivamente, por lo tanto, la vía conformista ya no tiene sentido, aumentando cada día la cantidad de personas que buscan ser sujetos por la vía contestataria o marginal, lo cual da origen a múltiples subculturas juveniles que buscan expresarse al interior de la escuela. 
Por otra parte, los individuos escapan a las formas instituidas de contestación y marginalidad, intentando fundar sus proyectos personales en principios culturales nuevos. El ser sujeto por la vía realista (del conformismo y de la movilidad social) ahora toma la forma de una reafirmación de valores del pasado. Es así como cada vez más jóvenes forman parte de una situación de aculturación: ellos no pueden adherir al antiguo modelo cultural porque lo encuentran indeseable e impracticable, pero -a la vezno pueden adherir al nuevo, dado que su legitimidad aún no está asegurada, por lo tanto, se ven obligados a tratar de conciliar los dos modelos en sus prácticas cotidianas.

Este profundo cambio cultural (mutación) se expresa en la diversidad de las subculturas juveniles, las que, entre otros espacios, se manifiesta en la escuela generando tensiones, problemas y conflictos difíciles de resolver.

Por una parte la escuela es portadora de una cultura organizacional e institucional formal, basada en tradiciones, estatutos, leyes y preceptos que se deben respetar para "jugar el juego" al interior de los espacios escolares; y por otra, los jóvenes son portadores de una diversidad cultural (subculturas) que se manifiesta en las llamadas tribus urbanas.

\section{Las tribus urbanas y la Escuela}

El fenómeno denominado Tribus Urbanas ha causado progresivo revuelo últimamente en nuestro país. Prueba de ello lo constituyen reiterados episodios de violencia juvenil suscitados en el Gran Santiago

Según datos de la SIP de Carabineros, existen alrededor de 18 "pandillas" juveniles en el Gran Santiago, concentradas principalmente en las comunas de San Miguel, Pedro Aguirre Cerda, Nuñoa, Recoleta, Conchalí y la Pintana. Entre las agrupaciones más renombradas podemos encontrar a los Sombra Latina de Maipú; los CNI, los RAS (resistencia anti social) y los MS (mente 
sucia) de San Miguel; los MJ (malas juntas) y los MC (mala clase) de Pedro Aguirre Cerda; los Fleming Clan de las Condes; los de la Villa Olímpica y los de la Villa Frei de Nuñoa; los Peñi y los Pinreb (Pintana rebelde) de la Pintana.

Las estadísticas señalan que los jóvenes que participan de estos grupos "en su gran mayoría son menores de dieciocho años, caracterizados como sujetos jóvenes, de procedencia marginal o de clase socioeconómica baja, con escasa o nula educación y que actúan en pandillas que fomentan la violencia y el delito, y tienden a causar mayor daño a sus víctimas" (Fundación Paz Ciudadana, 1997, No 5 ).

Frente a este fenómeno, la opinión pública ha mostrado un creciente nivel de preocupación, pero no se cuenta -en este momento- con una batería interpretativa de la problemática que contribuya a caracterizar y entender en profundidad el fenómeno. En ese sentido, las escasas aproximaciones a este tipo de dinámicas juveniles provienen -generalmente- del discurso dominante que existe en torno a estos microgrupos, vale decir, de las indagaciones policiales o de los medios de comunicación, donde la tendencia es a encapsularlos bajo el rótulo y el estigma de la delincuencia, la drogadicción, la violencia y las bandas juveniles (eje de la desadaptación y la desviación social).

No obstante, la emergencia y proliferación de las Tribus Urbanas se deja comprender mucho más eficazmente cuando las consideramos como la expresión de prácticas sociales y culturales más soterradas, que de un modo u otro están dando cuenta de una época vertiginosa y en constante proceso de mutación cultural y recambio de sus imaginarios simbólicos. Proceso que incluso comienza a minar las categorías con las cuales cuentan las ciencias sociales para abordar la complejidad social, y que particularmente en el caso de las nociones ligadas a la juventud la realidad parece desbordar más rápidamente los conceptos con los que se trabaja. Por lo cual se hace necesario y urgente generar 
una aproximación reflexiva encaminada a superar dichos desajustes.

La sensibilidad juvenil actual comienza a poner en práctica toda una ritualidad distintiva, que va marcando y protegiendo el espacio de su cotidianeidad. Conjuntamente con ello se va produciendo una resignificación del hábitat urbano donde se desenvuelve esta sensibilidad. "Ésta, se caracteriza por un "devenir" que va desde la periferia al (un) centro y que muchas veces es sin rumbo definido. Es el "andar carreteando", donde el énfasis está puesto en el "andar" (...) En ese deambular, el encuentro con un otro mediado por las "marcas", facilita el identificarse. Son las señas de reconocimiento que les permite catalogarse como: hippie, thrasher, punki, cuico, artesa, tecno, under, etc. En este "andar" los jóvenes se reconocen diversos, se re-encuentran en el contraste; en la diferencia que, si es respetada, exige la contraparte" (Soto. P., 1994. pp. 44 - 45).

En este último sentido, las Tribus Urbanas podrían constituir una cristalización de tensiones, encrucijadas y ansiedades que atraviesan a la(s) juventud(es) contemporánea(s). Son la expresión de una crisis de sentido a la cual nos arroja la modernidad, pero también constituyen la manifestación de una disidencia cultural o una "resistencia" ante una sociedad desencantada por la globalización del proceso de racionalización, la masificación y la inercia que caracteriza la vida en las urbes hipertrofiadas, donde todo parece correr en función del éxito personal y el consumismo alienante.

Estas tribus urbanas con sus respectivas subculturas se infiltran en los espacios escolares buscando escenarios donde manifestarse, donde ser reconocidos y respetados, sin embargo, pareciera ser que la cultura institucional de los espacios escolares no está preparada para recibirlos y legitimarlos en sus manifestaciones y prácticas simbólicas; lo que se aprecia es más bien el rechazo, la molestia o la indiferencia frente a este tipo de manifestaciones culturales juveniles. 
Frente a este proceso, las Tribus Urbanas son la instancia para intensificar la experiencia biográfica y la afectividad colectiva, el contacto humano y sobre todo la alternativa de construir identidad y potenciar una imagen social. En otras palabras, las Tribus Urbanas constituyen una posibilidad de recrear una nueva "socialidad", de reeditar un nuevo orden simbólico a partir del tejido social cotidiano. Pero, sobre este punto los medios de comunicación también juegan un rol preponderante, en tanto combustionan el proceso de tribalización actual: los reportajes, la moda, el cine, la música, etc. Lo que lleva a inferir una especie de alianza tácita entre medios de comunicación y Tribus. Asociación que no deja de ser contradictoria: los medios demonizan pero simultáneamente fortalecen su desarrollo.

En suma, hasta ahora las ciencias sociales han puesto el énfasis en un discurso oficial/institucional para explicarse las tensiones de sentido por las cuales atraviesa la sociedad chilena y particularmente la realidad juvenil, lo que dificulta una lectura plural de estas tensiones y la cultura escolar se encuentra atrapada en este dilema. De este modo, una lectura heterónoma necesariamente debe explorar en las narrativas informales, donde se modulan los recursos de expresión simbólica de memorias y subjetividades en ambiguos conflictos de representación. Bajo este supuesto una nueva mirada a este tipo de cultura juvenil debe explorar y rescatar la praxis discursiva presente en estas agrupaciones, y que de un modo u otro refiere simultáneamente a un tipo de saber específico/cotidiano y a determinadas lógicas comportamentales que se constituyen al interior de estas nuevas formas de asociación juvenil -las Tribus Urbanas-.

Todo ello con el propósito de contribuir no sólo a generar un proceso de des-estigmatización de este tipo de jóvenes en nuestra sociedad, sino que por sobre todo a instalar un enfoque pluralista que ayude a comprender más integralmente las problemáticas y las realidades propias del mundo juvenil actual. Esta comprensión es vital para los profesores; son ellos quienes cotidianamente 
se relacionan con los jóvenes y conocen fácticamente estas expresiones culturales, ellos (los profesores) son tensionados a aceptar formas de expresión que no conocen y no re-conocen dado que la mayoría de las veces se parapetan en la cultural institucional, apelando a los grandes conceptos de orden y disciplina; conceptos sin los cuales no se puede administrar el espacio escolar.

El tiempo actual se presenta para una gran cantidad de jóvenes en nuestro país, como un tiempo de incertidumbre y de inseguridad. Es un tiempo de crisis, el cual se puede caracterizar por conceptos que intentan ser parámetros tales como: globalización, mutaciones culturales, hibridizaciones, etc. En suma, podemos decir crisis de adaptaciones sociales; especialmente asociadas a los campos de la economía, la educación, las comunicaciones y la ética en las relaciones humanas cotidianas e institucionales, públicas y privadas, en un contexto de modernidad periférica (Sarlo B., 1988), los cuales serían rasgos reveladores de esta condición.

Esta crisis es vivida profundamente en distintos planos y constituiría el actual estado que experimenta la sociedad modernizada o en vías de modernización, como efecto progresivo de los procesos de secularización y racionalización, con el efecto, postulado por Weber, de desencantamiento del mundo. Abstrayendo otras dimensiones, sin duda de gran importancia, podemos decir que la modernidad y la secularización como contexto societal en un medio hibridizado (García N., 1995) en los hechos, va desintegrando y /o mutando una visión de mundo y sus distintos órdenes institucionales, mutación que se manifiesta fuertemente en el ámbito de lo cultural, caracterizándose, siguiendo a Franssen, (1994) por los procesos de "mutación cultural" los cuales se pueden visualizar "por la importancia creciente de las industrias culturales (medios de comunicación de masas) y tiene implicaciones no solamente en cuanto a los bienes culturales y a los códigos necesarios para su consumo, sino también en el sentido mismo de la experiencia de los individuos" (Franssen, A. 1994, p.15) 
Esta cuestión de fondo, que se extiende al plano de los comportamientos juveniles escolares donde, según algunos autores, se observa un proceso paulatino de rechazo al valor intrínseco de las normas y sus supuestos y/o su aceptación instrumental en función de objetivos inmediatos, lo que permite construir imágenes de los jóvenes, etiquetándolos como: "individualistas", "consumistas", "amorales", "apolíticos", entre otros,

Hace algunas décadas la juventud chilena fue caracterizada por algunos enfoques teóricos predominantes (Valenzuela E., 1985), como una juventud "anómica" y desintegrada que expresaba efectos y cambios socioculturales supuestamente no deseados de la socialización en el proceso de modernización que estaba viviendo el país. Se hacía referencia a la crisis de adaptación e integración expresada en la desarticulación del mundo colectivo y a la crisis de identidad cultural que se experimentaba en la desarticulación de los valores, expresada, en la desintegración de la comunidad y una ruptura de las relaciones primarias.

Desde esos trabajos la anomia se visualiza como la emergencia de los deseos y las pasiones: vivir el inmediatismo a través de la evasión o la agresión y simultáneamente vivir el inconformismo. Una lectura que extrema la definición sociológica de anomia de Durkheim, respecto de la inexistencia relativa de normas, al extremo del caos. Esta experiencia escaparía a cualquier control normativo, manifestándose especialmente en la juventud. Lo anterior es especialmente claro en el encuentro que se produce a diario entre los jóvenes estudiantes y las normas escolares. Muchos jóvenes desconocen estas normas o intentan burlarlas permanentemente.

Esta lectura extremista adquiere algún grado de moderación cuando se replantea la concepción de anomia desde la perspectiva funcionalista mertoniana, entendida como modos de adaptación para alcanzar fines institucionalmente sancionados y valorados, por medios también institucionalmente sancionados y valorados 
-en la mayor parte de los casos tipificados por el propio Merton (Merton, 1972)-. Hablamos entonces de crisis de adaptación en el marco de transformaciones y cambios socioculturales inevitables en el marco de la modernidad.

Este enfoque proveniente de lo que se ha denominado la sociología estructural-funcionalista (eje de la desviación social), se inicia a partir de los estudios de Ralph Linton (1942), quién "observando" a los adolescentes norteamericanos en los colegios (High School), se va dando cuenta, que éstos, están comenzando a construir un mundo separado al de sus propios padres con sus propias normas y valores. La escuela comienza a transformarse en el centro de la vida social de los jóvenes, en un espacio que origina una nueva sociabilidad y una lógica propia.

Posteriormente otro autor central de esta corriente, Parsons, desarrollará en profundidad estas ideas, legitimando el surgimiento de una "cultura juvenil", cultura que generaba una nueva conciencia generacional, que "cristalizaba en una cultura autónoma e interclasista centrada en el consumo hedonista", a pesar que ésta no producía, por estar todavía en el aparato educativo. Esto lleva a Parsons a señalar, que la cultura juvenil se aleja cada vez más del trabajo e incluso de la estructura de clases, ya que el acceso al tiempo libre por ejemplo, parece cancelar las diferencias sociales entre los jóvenes, uniformándose la cultura juvenil, en la medida en que se vinculan al mercado a través del consumo.

Se puede plantear, a manera de crítica, especialmente al modelo funcionalista, que la situación de los jóvenes en la sociedad no puede ser reducida a un mecanismo de integración funcional, sino que se requiere el reconocimiento de la existencia de un sujeto particular que se identificaría con orientaciones culturales generales y con convicciones personales y colectivas ligadas a su propio quehacer.

A partir de esto, este sujeto-joven abierto a los procesos, enfrentado al fenómeno de la modernidad, queda expuesto a una 
serie de situaciones: la absorción por la imagen o la exclusión sin salida (Tijoux, 1993); la internalización de los signos de muerte como valores propios o la búsqueda de una identidad que de cuenta de las expectativas, valores y sueños (Duarte, 1994); tomar una actitud de total pragmatismo con la realidad (atinar) o fundar un nuevo mundo (adanismo) ; asumir conductas colectivas e individuales que se expresan a través de mecanismos de agresión, compensación y resignación, para estar ahí, para tener una ilusión de participar, porque eso es lo que los hace sentirse virtualmente integrados en medio de la exclusión real, por falta de capacidad real de compra.

\section{Conclusiones}

Como hemos podido ver, la escuela juega un rol central en la mayoría de los jóvenes de nuestro país, rol que trasciende el ámbito específicamente educativo, constituyéndose en un potente agente socializador; de esta manera la disciplina sociológica contribuye al trabajo educativo puesto que colabora en los procesos de administración educacional y en los procesos de enseñanzaaprendizaje que viven los jóvenes.

Es así como un número importante de actividades escolares refleja la contribución sociológica a las áreas de desarrollo juvenil (mencionadas anteriormente), al proceso de aprendizaje, a la comunidad escolar y al cambio social.

Para comprender al mundo juvenil y sus comportamientos al interior de los espacios escolares es necesario desarrollar nuevos enfoques analíticos, enfoques que opten por la perspectiva de la construcción de sujetos, permitiendo que ellos mismos se caractericen 'como jóvenes de una nueva época', 'de otra era', o 'que están en otra', asumiendo que ese "estar en otra" a que hacen referencia evoca un momento y un espacio determinado que tiene características propias, diferente de los adultos, a sus sistemas de vida, a la autoridad, y a todo aquello que represente los modos tradicionales de la vida social, entre ellos, la escuela. 
La posibilidad de desarrollar una educación de calidad no radica solamente en disponer de adecuadas infraestructuras o de incorporar las TICs a los procesos educativos, sino que pasa por la formación permanente de los docentes en los contenidos de la llamada cultura juvenil. Es preciso conocer a los jóvenes en profundidad porque solo de esa manera se podrá ser asertivos en su educación.

Los contenidos y metodologías de enseñanza-aprendizaje deben incorporar a los jóvenes como sujetos activos del proceso y para lograrlo hay que escuchar, aceptar y acoger a los jóvenes en toda su complejidad, de lo contrario las subculturas juveniles que viven al interior de los establecimientos escolares serán un constante problema por resolver o un obstáculo que salvar y no un eficaz aliado en pro de objetivos comunes; en síntesis se trata de concretar lo que ya el Mece-Media planteaba hace algunos años atrás: que los jóvenes sean co-constructores de su educación y para lograrlo en primer lugar hay que conocerlos y luego incorporarlos activamente en los procesos educativos.

\section{Bibliografía}

Alpízar, Lydia \& Bernal, Marina (2003). "La construcción social de las juventudes". En Revista Última Década - (pp. 105-124). CIDPA.

Bajoit, Guy \& Franssen, Abraham (1995). Les Jeunes dans la Compétition Culturelle, Sociologie d'aujoud'hui. PUF.

Barbero, Jesús (2001). "Desafíos del País a la juventud y de la juventud al País". Desde la Región 33, Mayo. Medellín, Colombia - www. region.org.co

Bourdieu, P. (2003). Capital cultural, escuela y espacio social. Siglo Veintiuno Editores.

Dewey, Jhon (1967). “La concepción democrática en educación". En Democracia y Educación. Editorial LOSADA, Buenos Aires.

Duarte, Klaudio (2002). Mundos jóvenes, mundos adultos: lo generacional y las posibilidades de reconstruir puentes rotos en el Liceo. En Revista Última Década. CIDPA. 
Dubet, F. \& Martucelli, D. (1998). En la escuela. Sociología de la experiencia escolar. Editorial LOSADA.

Franssen, A (1994). Los jóvenes secundarios en la mutación cultural: Jenny, Carola y Mauricio. CIDE, Doc. $\mathrm{N}^{\mathrm{o}} 4$.

Fundación Paz Ciudadana (1997). Conceptos para la prevención y contención del delito, $\mathrm{N}^{\circ} 5$ y N $\mathrm{N}^{\mathrm{0}} 11$, Santiago de Chile.

Garcés, Ángela (2006). “Juventud y escuela. Percepciones y estereotipos que rondan el espacio escolar". En Revista Última Década. N² 24.

García Canclini, N. (1995). Culturas Híbridas. Ed. Sudamericana, Buenos. Aires.

Oyarzún, A. et. al. (2001). Entre jóvenes re-productores y jóvenes co-constructores: Sentidos de la integración en la cultura escolar. CIDPA Ediciones. Marzo.

Sarlo, Beatriz (1988). Una Modernidad Periférica. Editorial Nueva Visión, Buenos. Aires.

Soto, P. (1994). "Suicidio Juvenil: características y significados asociados". Silencio, cansancio, derrota (pp. 44-45), Instituto Nacional de la Juventud.

Tijoux, María Emilia (1998). Jóvenes en la calle: realidad de la Región Metropolitana. Univercidad ARCIS. Santiago de Chile.

Valenzuela, E (1985). La rebelión de los jóvenes. SUR, Santiago de Chile. 